\title{
A new extension of a Hardy-Hilbert-type inequality
}

Qiliang Huang*

"Correspondence:
qlhuang@yeah.net
Department of Mathematics,
Guangdong University of
Education, Guangzhou, Guangdong
510303, P.R. China

"Correspondence: qlhuang@yeah.net Department of Mathematics, 510303, P.R. China

\begin{abstract}
By introducing independent parameters, and applying weight coefficients and the technique of real analysis, we give a new extension of a Hardy-Hilbert-type inequality with a best possible constant factor. Furthermore, the equivalent forms, the operator expressions, and the reverses are considered.
\end{abstract}

MSC: 26D15; 47A07

Keywords: Hardy-Hilbert-type inequality; parameter; weight coefficient; equivalent form; reverse

\section{Introduction}

If $p>1, \frac{1}{p}+\frac{1}{q}=1, a_{n}, b_{n} \geq 0,0<\sum_{n=1}^{\infty} a_{n}^{p}<\infty$ and $0<\sum_{n=1}^{\infty} b_{n}^{q}<\infty$, then we have the Hardy-Hilbert inequality as follows (cf. [1]):

$$
\sum_{n=1}^{\infty} \sum_{m=1}^{\infty} \frac{a_{m} b_{n}}{m+n}<\frac{\pi}{\sin (\pi / p)}\left(\sum_{n=1}^{\infty} a_{n}^{p}\right)^{1 / p}\left(\sum_{n=1}^{\infty} b_{n}^{q}\right)^{1 / q},
$$

where the constant factor $\frac{\pi}{\sin (\pi / p)}$ is the best possible. We also have the following HardyHilbert-type inequality $(c f .[2])$ :

$$
\sum_{n=1}^{\infty} \sum_{m=1}^{\infty} \frac{\ln (m / n) a_{m} b_{n}}{m-n}<\left[\frac{\pi}{\sin (\pi / p)}\right]^{2}\left(\sum_{n=1}^{\infty} a_{n}^{p}\right)^{1 / p}\left(\sum_{n=1}^{\infty} b_{n}^{q}\right)^{1 / q},
$$

where the constant factor $\left[\frac{\pi}{\sin (\pi / p)}\right]^{2}$ is still the best possible. In 2008, by introducing some parameters, Yang gave an extension of inequality (2) (cf. [3]): If $0<\lambda_{1}, \lambda_{2} \leq 1, \lambda_{1}+\lambda_{2}=\lambda$, $a_{n}, b_{n} \geq 0,0<\sum_{n=1}^{\infty} n^{p\left(1-\lambda_{1}\right)-1} a_{n}^{p}<\infty$, and $0<\sum_{n=1}^{\infty} n^{q\left(1-\lambda_{2}\right)-1} b_{n}^{q}<\infty$, then the following inequality holds:

$$
\begin{aligned}
& \sum_{n=1}^{\infty} \sum_{m=1}^{\infty} \frac{\ln (m / n) a_{m} b_{n}}{m^{\lambda}-n^{\lambda}} \\
& \quad<\left[\frac{\pi}{\lambda \sin \left(\pi \lambda_{1} / \lambda\right)}\right]^{2}\left(\sum_{n=1}^{\infty} n^{p\left(1-\lambda_{1}\right)-1} a_{n}^{p}\right)^{1 / p}\left(\sum_{n=1}^{\infty} n^{q\left(1-\lambda_{2}\right)-1} b_{n}^{q}\right)^{1 / q},
\end{aligned}
$$

(c) 2015 Huang. This article is distributed under the terms of the Creative Commons Attribution 4.0 International License (http://creativecommons.org/licenses/by/4.0/), which permits unrestricted use, distribution, and reproduction in any medium, provided you give appropriate credit to the original author(s) and the source, provide a link to the Creative Commons license, and indicate if changes were made. 
where the constant factor $\left[\frac{\pi}{\lambda \sin \left(\pi \lambda_{1} / \lambda\right)}\right]^{2}$ is the best possible. There are lots of improvements, generalizations, and applications of inequality (2) ([3-11]). For more details, Yang gives a summary of introducing independent parameters $([12,13])$.

In this article, by introducing independent parameters, and applying weight coefficients and the technique of real analysis, we give a new extension of (2) with a best possible constant factor. Furthermore, the equivalent forms, the operator expressions, and the reverses are considered.

\section{Some lemmas}

We agree on the following assumptions in this paper: $p \neq 0, \frac{1}{p}+\frac{1}{q}=1, \lambda>0,0<\lambda_{i} \leq 1$ $(i=1,2), \lambda_{1}+\lambda_{2}=\lambda, k_{\lambda}\left(\lambda_{2}\right)=k_{\lambda}\left(\lambda_{1}\right)=\left[\frac{\pi}{\lambda \sin \left(\pi \lambda_{1} / \lambda\right)}\right]^{2},\left\{\mu_{m}\right\}_{m=1}^{\infty}$ and $\left\{v_{n}\right\}_{n=1}^{\infty}$ are positive sequences, $U_{m}=\sum_{i=1}^{m} \mu_{i}, V_{n}=\sum_{i=1}^{n} v_{i}$, and $a_{n}, b_{n} \geq 0(m, n \in \mathbf{N}=\{1,2, \ldots\})$,

$$
0<\sum_{m=1}^{\infty} \frac{U_{m}^{p\left(1-\lambda_{1}\right)-1}}{\mu_{m}^{p-1}} a_{m}^{p}<\infty, \quad 0<\sum_{n=1}^{\infty} \frac{V_{n}^{q\left(1-\lambda_{2}\right)-1}}{v_{n}^{q-1}} b_{n}^{q}<\infty .
$$

Lemma 1 Define the weight coefficients as follows:

$$
\begin{aligned}
& \omega\left(\lambda_{2}, m\right):=\sum_{n=1}^{\infty} \frac{\ln \left(U_{m} / V_{n}\right)}{U_{m}^{\lambda}-V_{n}^{\lambda}} \frac{U_{m}^{\lambda_{1}}}{V_{n}^{1-\lambda_{2}}} v_{n}, \quad m \in \mathbf{N}, \\
& \varpi\left(\lambda_{1}, n\right):=\sum_{m=1}^{\infty} \frac{\ln \left(U_{m} / V_{n}\right)}{U_{m}^{\lambda}-V_{n}^{\lambda}} \frac{V_{n}^{\lambda_{2}}}{U_{m}^{1-\lambda_{1}}} \mu_{m}, \quad n \in \mathbf{N} .
\end{aligned}
$$

We have the following inequalities:

$$
\begin{array}{ll}
\omega\left(\lambda_{2}, m\right)<k_{\lambda}\left(\lambda_{1}\right) & \left(m \in \mathbf{N} ; 0<\lambda_{2} \leq 1, \lambda_{1}>0\right), \\
\varpi\left(\lambda_{1}, n\right)<k_{\lambda}\left(\lambda_{1}\right) & \left(n \in \mathbf{N} ; 0<\lambda_{1} \leq 1, \lambda_{2}>0\right) .
\end{array}
$$

Proof Putting $\mu(t):=\mu_{m}, t \in(m-1, m](m=1,2, \ldots), v(t):=v_{n}, t \in(n-1, n](n=1,2, \ldots)$,

$$
U(x):=\int_{0}^{x} \mu(t) d t \quad(x \geq 0), \quad V(y):=\int_{0}^{y} v(t) d t \quad(y \geq 0) .
$$

Then we have $U(m)=U_{m}, V(n)=V_{n}(m, n \in \mathbf{N}) \cdot U^{\prime}(x)=\mu(x)=\mu_{m}$ when $x \in(m-1, m]$; $V^{\prime}(y)=v(y)=v_{n}$ when $y \in(n-1, n]$. Since the function $V(y)(y>0)$ is strictly increasing and $f(x)=\frac{\ln (m / x)}{m^{\lambda}-x^{\lambda}}(x>0)$ is strictly decreasing (cf. [4], Example 2.2.1), in view of $1-\lambda_{2} \geq 0$, we have

$$
\begin{aligned}
\omega\left(\lambda_{2}, m\right) & =\sum_{n=1}^{\infty} \int_{n-1}^{n} \frac{\ln \left(U_{m} / V_{n}\right)}{U_{m}^{\lambda}-V_{n}^{\lambda}} \frac{U_{m}^{\lambda_{1}}}{V_{n}^{1-\lambda_{2}}} V^{\prime}(t) d t \\
& <\sum_{n=1}^{\infty} \int_{n-1}^{n} \frac{\ln \left(U_{m} / V(t)\right)}{U_{m}^{\lambda}-V^{\lambda}(t)} \frac{U_{m}^{\lambda_{1}}}{V^{1-\lambda_{2}}(t)} V^{\prime}(t) d t .
\end{aligned}
$$

Putting $u=\frac{V^{\lambda}(t)}{U_{m}^{\lambda}}$ in the above integral, and in view of the fact that (cf. [2])

$$
\int_{0}^{\infty} \frac{\ln u}{u-1} u^{a-1} d u=\left[\frac{\pi}{\sin (a \pi)}\right]^{2} \quad(0<a<1)
$$


it follows that

$$
\begin{aligned}
\omega\left(\lambda_{2}, m\right) & <\frac{1}{\lambda^{2}} \sum_{n=1}^{\infty} \int_{\frac{V^{\lambda}(n-1)}{u_{m}^{\lambda}}}^{\frac{V^{\lambda}(n)}{\lambda}} \frac{\ln u}{u-1} u^{\frac{\lambda_{2}}{\lambda}-1} d u \\
& =\frac{1}{\lambda^{2}} \int_{0}^{\frac{V^{\lambda}(\infty)}{u_{m}^{\lambda}}} \frac{\ln u}{u-1} u^{\frac{\lambda_{2}}{\lambda}-1} d u \leq \frac{1}{\lambda^{2}} \int_{0}^{\infty} \frac{\ln u}{u-1} u^{\frac{\lambda_{2}}{\lambda}-1} d u \\
& =\left[\frac{\pi}{\lambda \sin \left(\pi \lambda_{2} / \lambda\right)}\right]^{2}=\left[\frac{\pi}{\lambda \sin \left(\pi \lambda_{1} / \lambda\right)}\right]^{2}=k_{\lambda}\left(\lambda_{1}\right) .
\end{aligned}
$$

Hence we prove that (6) is valid. In the same way, we can prove that (7) is valid too.

Lemma 2 Suppose that $\left\{\mu_{m}\right\}_{m=1}^{\infty}$ and $\left\{v_{n}\right\}_{n=1}^{\infty}$ are decreasing sequences, and $U(\infty)=$ $V(\infty)=\infty$, then we have the following inequalities:

$$
\begin{aligned}
& k_{\lambda}\left(\lambda_{1}\right)\left(1-\theta_{1}\left(\lambda_{2}, m\right)\right)<\omega\left(\lambda_{2}, m\right) \quad\left(m \in \mathbf{N} ; 0<\lambda_{2} \leq 1, \lambda_{1}>0\right), \\
& k_{\lambda}\left(\lambda_{1}\right)\left(1-\theta_{2}\left(\lambda_{1}, n\right)\right)<\varpi\left(\lambda_{1}, n\right) \quad\left(n \in \mathbf{N} ; 0<\lambda_{1} \leq 1, \lambda_{2}>0\right),
\end{aligned}
$$

where $\theta_{1}\left(\lambda_{2}, m\right)=O\left(\frac{1}{u_{m}^{\lambda_{2} / 2}}\right) \in(0,1)$ and $\theta_{2}\left(\lambda_{1}, n\right)=O\left(\frac{1}{V_{n}^{\lambda_{1} / 2}}\right) \in(0,1)$. Moreover, we get

$$
\begin{aligned}
& \sum_{m=1}^{\infty} \frac{\mu_{m}}{U_{m}^{1+\varepsilon}}=\frac{1}{\varepsilon}\left(1+o_{1}(1)\right) \quad\left(\varepsilon \rightarrow 0^{+}\right), \\
& \sum_{n=1}^{\infty} \frac{v_{n}}{V_{n}^{1+\varepsilon}}=\frac{1}{\varepsilon}\left(1+o_{2}(1)\right) \quad\left(\varepsilon \rightarrow 0^{+}\right) .
\end{aligned}
$$

Proof By the decreasing property of $\left\{v_{n}\right\}_{n=1}^{\infty}$, and in view of $1-\lambda_{2} \geq 0, V(\infty)=\infty$, we find

$$
\begin{aligned}
\omega\left(\lambda_{2}, m\right) & \geq \sum_{n=1}^{\infty} \frac{\ln \left(U_{m} / V_{n}\right)}{U_{m}^{\lambda}-V_{n}^{\lambda}} \frac{U_{m}^{\lambda_{1}}}{V_{n}^{1-\lambda_{2}}} v_{n+1} \\
& =\sum_{n=1}^{\infty} \int_{n}^{n+1} \frac{\ln \left(U_{m} / V_{n}\right)}{U_{m}^{\lambda}-V_{n}^{\lambda}} \frac{U_{m}^{\lambda_{1}}}{V_{n}^{1-\lambda_{2}}} V^{\prime}(t) d t \\
& >\sum_{n=1}^{\infty} \int_{n}^{n+1} \frac{\ln \left(U_{m} / V(t)\right)}{U_{m}^{\lambda}-V^{\lambda}(t)} \frac{U_{m}^{\lambda_{1}}}{V^{1-\lambda_{2}}(t)} V^{\prime}(t) d t \\
& =\frac{1}{\lambda^{2}} \sum_{n=1}^{\infty} \int_{\frac{V^{\lambda}(n)}{u_{m}^{\lambda}}}^{\frac{V^{\lambda}(n+1)}{u_{m}^{\lambda}}} \frac{\ln u}{u-1} u^{\frac{\lambda_{2}}{\lambda}-1} d u=\frac{1}{\lambda^{2}} \int_{\frac{V^{\lambda}(1)}{U_{m}^{\lambda}}}^{\infty} \frac{\ln u}{u-1} u^{\frac{\lambda_{2}}{\lambda}-1} d u \\
& =k_{\lambda}\left(\lambda_{1}\right)-\frac{1}{\lambda^{2}} \int_{0}^{\frac{v_{1}^{\lambda}}{u_{m}^{\lambda}}} \frac{\ln u}{u-1} u^{\frac{\lambda_{2}}{\lambda}-1} d u=k_{\lambda}\left(\lambda_{1}\right)\left(1-\theta_{1}\left(\lambda_{2}, m\right)\right),
\end{aligned}
$$

where

$$
\theta_{1}\left(\lambda_{2}, m\right):=\frac{1}{\lambda^{2} k_{\lambda}\left(\lambda_{1}\right)} \int_{0}^{\frac{v_{1}^{\lambda}}{u_{m}^{\lambda}}} \frac{\ln u}{u-1} u^{\frac{\lambda_{2}}{\lambda}-1} d u \in(0,1)
$$


In virtue of

$$
\begin{aligned}
& \lim _{x \rightarrow \infty} \frac{\int_{0}^{\nu_{1}^{\lambda} / x^{\lambda}} \frac{\ln u}{u-1} u^{\frac{\lambda_{2}}{\lambda}-1} d u}{x^{-\lambda_{2} / 2}} \\
& =\lim _{x \rightarrow \infty} \frac{2 \lambda^{2} v_{1}^{\lambda_{2}}}{\lambda_{2}}\left(\frac{\nu_{1}^{\lambda}}{x^{\lambda}}-1\right)^{-1}\left(\frac{1}{x^{\lambda_{2} / 2}} \ln \frac{\nu_{1}}{x}\right)=0,
\end{aligned}
$$

it is obvious that $\theta_{1}\left(\lambda_{2}, m\right)=O\left(\frac{1}{u_{m}^{\lambda_{2} / 2}}\right)$. Hence (8) is valid. In the same way, we can prove that (9) is valid too. Moreover, we have

$$
\begin{aligned}
\sum_{m=1}^{\infty} \frac{\mu_{m}}{U_{m}^{1+\varepsilon}} & =\frac{1}{\mu_{1}^{\varepsilon}}+\sum_{m=2}^{\infty} \int_{m-1}^{m} \frac{U^{\prime}(t)}{U_{m}^{1+\varepsilon}} d t \\
& \leq \frac{1}{\mu_{1}^{\varepsilon}}+\sum_{m=2}^{\infty} \int_{m-1}^{m} \frac{U^{\prime}(t)}{U^{1+\varepsilon}(t)} d t \\
& =\frac{1}{\mu_{1}^{\varepsilon}}+\sum_{m=2}^{\infty} \int_{U(m-1)}^{U(m)} \frac{1}{u^{1+\varepsilon}} d u=\frac{1}{\mu_{1}^{\varepsilon}}+\int_{\mu_{1}}^{\infty} \frac{1}{u^{1+\varepsilon}} d u \\
& =\frac{1}{\varepsilon}\left[1+\left(\frac{1}{\mu_{1}^{\varepsilon}}+\frac{\varepsilon}{\mu_{1}^{\varepsilon}}-1\right)\right], \\
\sum_{m=1}^{\infty} \frac{\mu_{m}}{U_{m}^{1+\varepsilon}} & \geq \sum_{m=1}^{\infty} \int_{m}^{m+1} \frac{\mu_{m+1}}{U_{m}^{1+\varepsilon}} d t \\
& =\sum_{m=1}^{\infty} \int_{m}^{m+1} \frac{U^{\prime}(t)}{U_{m}^{1+\varepsilon}} d t>\sum_{m=1}^{\infty} \int_{m}^{m+1} \frac{U^{\prime}(t)}{U^{1+\varepsilon}(t)} d t \\
& =\sum_{m=1}^{\infty} \int_{U(m)}^{U(m+1)} \frac{1}{u^{1+\varepsilon}} d u=\int_{\mu_{1}}^{\infty} \frac{1}{u^{1+\varepsilon}} d u \\
& =\frac{1}{\varepsilon}\left[1+\left(\frac{1}{\mu_{1}^{\varepsilon}}-1\right)\right] .
\end{aligned}
$$

Then we have (10). In the same way, we have (11).

Remark 1 Taking $\varepsilon=a>0$, we write by (10) and (11) that

$$
\sum_{m=1}^{\infty} \frac{\mu_{m}}{U_{m}^{1+a}}=O_{1}(1), \quad \sum_{n=1}^{\infty} \frac{v_{n}}{V_{n}^{1+a}}=O_{2}(1) .
$$

\section{Equivalent forms and operator expressions}

Theorem 1 Suppose that $p>1$, then we have the following equivalent inequalities:

$$
\begin{aligned}
I: & : \sum_{n=1}^{\infty} \sum_{m=1}^{\infty} \frac{\ln \left(U_{m} / V_{n}\right)}{U_{m}^{\lambda}-V_{n}^{\lambda}} a_{m} b_{n} \\
& <\left[\frac{\pi}{\lambda \sin \left(\pi \lambda_{1} / \lambda\right)}\right]^{2}\left[\sum_{m=1}^{\infty} \frac{U_{m}^{p\left(1-\lambda_{1}\right)-1}}{\mu_{m}^{p-1}} a_{m}^{p}\right]^{1 / p}\left[\sum_{n=1}^{\infty} \frac{V_{n}^{q\left(1-\lambda_{2}\right)-1}}{v_{n}^{q-1}} b_{n}^{q}\right]^{1 / q},
\end{aligned}
$$




$$
\begin{aligned}
J & :=\left\{\sum_{n=1}^{\infty} \frac{v_{n}}{V_{n}^{1-p \lambda_{2}}}\left(\sum_{m=1}^{\infty} \frac{\ln \left(U_{m} / V_{n}\right)}{U_{m}^{\lambda}-V_{n}^{\lambda}} a_{m}\right)^{p}\right\}^{\frac{1}{p}} \\
& <\left[\frac{\pi}{\lambda \sin \left(\pi \lambda_{1} / \lambda\right)}\right]^{2}\left(\sum_{m=1}^{\infty} \frac{U_{m}^{p\left(1-\lambda_{1}\right)-1}}{\mu_{m}^{p-1}} a_{m}^{p}\right)^{1 / p} .
\end{aligned}
$$

Proof By Hölder's inequality with weight (cf. [14]), we find

$$
\begin{aligned}
& \left(\sum_{m=1}^{\infty} \frac{\ln \left(U_{m} / V_{n}\right)}{U_{m}^{\lambda}-V_{n}^{\lambda}} a_{m}\right)^{p} \\
& \quad=\left\{\sum_{m=1}^{\infty} \frac{\ln \left(U_{m} / V_{n}\right)}{U_{m}^{\lambda}-V_{n}^{\lambda}}\left[\frac{U_{m}^{\left(1-\lambda_{1}\right) / q} v_{n}^{1 / p}}{V_{n}^{\left(1-\lambda_{2}\right) / p} \mu_{m}^{1 / q}} a_{m}\right]\left[\frac{V_{n}^{\left(1-\lambda_{2}\right) / p} \mu_{m}^{1 / q}}{U_{m}^{\left(1-\lambda_{1}\right) / q} v_{n}^{1 / p}}\right]\right\}^{p} \\
& \quad \leq \sum_{m=1}^{\infty} \frac{\ln \left(U_{m} / V_{n}\right)}{U_{m}^{\lambda}-V_{n}^{\lambda}} \frac{U_{m}^{\left(1-\lambda_{1}\right) p / q} v_{n}}{V_{n}^{1-\lambda_{2}} \mu_{m}^{p / q}} a_{m}^{p}\left[\sum_{m=1}^{\infty} \frac{\ln \left(U_{m} / V_{n}\right)}{U_{m}^{\lambda}-V_{n}^{\lambda}} \frac{V_{n}^{\left(1-\lambda_{2}\right)(q-1)} \mu_{m}}{U_{m}^{1-\lambda_{1}} v_{n}^{q-1}}\right]^{p-1} \\
& \quad=\left(\varpi\left(\lambda_{1}, n\right)\right)^{p-1} \frac{V_{n}^{1-p \lambda_{2}}}{v_{n}} \sum_{m=1}^{\infty} \frac{\ln \left(U_{m} / V_{n}\right)}{U_{m}^{\lambda}-V_{n}^{\lambda}} \frac{U_{m}^{\left(1-\lambda_{1}\right) p / q} v_{n}}{V_{n}^{1-\lambda_{2}} \mu_{m}^{p / q}} a_{m}^{p} .
\end{aligned}
$$

By (7), it follows that

$$
\begin{aligned}
J & <\left(k_{\lambda}\left(\lambda_{1}\right)\right)^{\frac{1}{q}}\left[\sum_{n=1}^{\infty} \sum_{m=1}^{\infty} \frac{\ln \left(U_{m} / V_{n}\right)}{U_{m}^{\lambda}-V_{n}^{\lambda}} \frac{U_{m}^{\left(1-\lambda_{1}\right) p / q} v_{n}}{V_{n}^{1-\lambda_{2}} \mu_{m}^{p / q}} a_{m}^{p}\right]^{\frac{1}{p}} \\
& =\left(k_{\lambda}\left(\lambda_{1}\right)\right)^{\frac{1}{q}}\left[\sum_{m=1}^{\infty} \sum_{n=1}^{\infty} \frac{\ln \left(U_{m} / V_{n}\right)}{U_{m}^{\lambda}-V_{n}^{\lambda}} \frac{U_{m}^{\left(1-\lambda_{1}\right)(p-1)} v_{n}}{V_{n}^{1-\lambda_{2}} \mu_{m}^{p-1}} a_{m}^{p}\right]^{\frac{1}{p}} \\
& =\left(k_{\lambda}\left(\lambda_{1}\right)\right)^{\frac{1}{q}}\left[\sum_{m=1}^{\infty} \omega\left(\lambda_{2}, m\right) \frac{U_{m}^{p\left(1-\lambda_{1}\right)-1}}{\mu_{m}^{p-1}} a_{m}^{p}\right]^{\frac{1}{p}} .
\end{aligned}
$$

Combining (8) and (15), we have (13).

Using Hölder's inequality again, we have

$$
\begin{aligned}
I & =\sum_{n=1}^{\infty}\left[\frac{v_{n}^{1 / p}}{V_{n}^{\frac{1}{p}-\lambda_{2}}} \sum_{m=1}^{\infty} \frac{\ln \left(U_{m} / V_{n}\right)}{U_{m}^{\lambda}-V_{n}^{\lambda}} a_{m}\right]\left[\frac{V_{n}^{\frac{1}{p}-\lambda_{2}}}{v_{n}^{1 / p}} b_{n}\right] \\
& \leq J\left[\sum_{n=1}^{\infty} \frac{V_{n}^{q\left(1-\lambda_{2}\right)-1}}{v_{n}^{q-1}} b_{n}^{q}\right]^{\frac{1}{q}},
\end{aligned}
$$

and then we have (12) by using (13). On the other hand, assuming that (12) is valid, setting

$$
b_{n}=\frac{v_{n}}{V_{n}^{1-p \lambda_{2}}}\left[\sum_{m=1}^{\infty} \frac{\ln \left(U_{m} / V_{n}\right)}{U_{m}^{\lambda}-V_{n}^{\lambda}} a_{m}\right]^{p-1}, \quad n \in \mathbf{N},
$$


then we find $J=\left[\sum_{n=1}^{\infty} \frac{V_{n}^{q\left(1-\lambda_{2}\right)-1}}{v_{n}^{q-1}} b_{n}^{q}\right]^{1 / p}$. By (15), it follows that $J<\infty$. If $J=0$, then (13) is trivially valid. If $0<J<\infty$, then we have

$$
\begin{aligned}
0 & <\sum_{n=1}^{\infty} \frac{V_{n}^{q\left(1-\lambda_{2}\right)-1}}{v_{n}^{q-1}} b_{n}^{q}=J^{p}=I \\
& <k_{\lambda}\left(\lambda_{1}\right)\left[\sum_{m=1}^{\infty} \frac{U_{m}^{p\left(1-\lambda_{1}\right)-1}}{\mu_{m}^{p-1}} a_{m}^{p}\right]^{\frac{1}{p}}\left[\sum_{n=1}^{\infty} \frac{V_{n}^{q\left(1-\lambda_{2}\right)-1}}{v_{n}^{q-1}} b_{n}^{q}\right]^{\frac{1}{q}}<\infty, \\
J & =\left[\sum_{n=1}^{\infty} \frac{V_{n}^{q\left(1-\lambda_{2}\right)-1}}{v_{n}^{q-1}} b_{n}^{q}\right]^{1 / p}<k_{\lambda}\left(\lambda_{1}\right)\left[\sum_{m=1}^{\infty} \frac{U_{m}^{p\left(1-\lambda_{1}\right)-1}}{\mu_{m}^{p-1}} a_{m}^{p}\right]^{\frac{1}{p}} .
\end{aligned}
$$

Hence (13) is valid, which is equivalent to (12).

Theorem 2 Suppose that $p>1,\left\{\mu_{m}\right\}_{m=1}^{\infty}$ and $\left\{v_{n}\right\}_{n=1}^{\infty}$ are decreasing positive sequences, and $U(\infty)=V(\infty)=\infty$, then the constant factor $k_{\lambda}\left(\lambda_{1}\right)=\left[\frac{\pi}{\lambda \sin \left(\lambda_{1} \pi / \lambda\right)}\right]^{2}$ is the best possible in (12) and (13).

Proof For $0<\varepsilon<p \lambda_{1}$, we set $\tilde{\lambda}_{1}=\lambda_{1}-\frac{\varepsilon}{p}(\in(0,1)), \tilde{\lambda}_{2}=\lambda_{2}+\frac{\varepsilon}{p}(>0), \widetilde{a}_{m}=U_{m}^{\widetilde{\lambda}_{1}-1} \mu_{m}, \widetilde{b}_{n}=$ $V_{n}^{\tilde{\lambda}_{2}-\varepsilon-1} v_{n}$. By (10), (11), and (9), in view of Remark 1, we find

$$
\begin{aligned}
& \sum_{m=1}^{\infty} \frac{U_{m}^{p\left(1-\lambda_{1}\right)-1}}{\mu_{m}^{p-1}} \widetilde{a}_{m}^{p}=\sum_{m=1}^{\infty} \frac{\mu_{m}}{U_{m}^{1+\varepsilon}}=\frac{1}{\varepsilon}\left(1+o_{1}(1)\right), \\
& \sum_{n=1}^{\infty} \frac{V_{n}^{q\left(1-\lambda_{2}\right)-1}}{v_{n}^{q-1}} \widetilde{b}_{n}^{q}=\sum_{n=1}^{\infty} \frac{v_{n}}{V_{n}^{1+\varepsilon}}=\frac{1}{\varepsilon}\left(1+o_{2}(1)\right), \\
& \widetilde{I}:=\sum_{n=1}^{\infty} \sum_{m=1}^{\infty} \frac{\ln \left(U_{m} / V_{n}\right)}{U_{m}^{\lambda}-V_{n}^{\lambda}} \widetilde{a}_{m} \widetilde{b}_{n} \\
& =\sum_{n=1}^{\infty}\left[\sum_{m=1}^{\infty} \frac{\ln \left(U_{m} / V_{n}\right)}{U_{m}^{\lambda}-V_{n}^{\lambda}} \frac{V_{n}^{\tilde{\lambda}_{2}} \mu_{m}}{U_{m}^{1-\widetilde{\lambda}_{1}}}\right] \frac{v_{n}}{V_{n}^{\varepsilon+1}} \\
& =\sum_{n=1}^{\infty} \varpi\left(\widetilde{\lambda}_{1}, n\right) \frac{v_{n}}{V_{n}^{\varepsilon+1}} \geq k_{\lambda}\left(\widetilde{\lambda}_{1}\right) \sum_{n=1}^{\infty}\left(1-\theta_{2}\left(\widetilde{\lambda}_{1}, n\right)\right) \frac{v_{n}}{V_{n}^{\varepsilon+1}} \\
& =k_{\lambda}\left(\widetilde{\lambda}_{1}\right)\left[\sum_{n=1}^{\infty} \frac{v_{n}}{V_{n}^{\varepsilon+1}}-\sum_{n=1}^{\infty} O\left(\frac{v_{n}}{\left.\left.V_{n}^{\frac{1}{2}\left(\frac{\varepsilon}{q}+\varepsilon+\lambda_{1}\right)+1}\right)\right]}\right.\right. \\
& =\frac{1}{\varepsilon}\left[\frac{\pi}{\lambda \sin \left(\pi \widetilde{\lambda}_{1} / \lambda\right)}\right]^{2}\left[1+o_{2}(1)-\varepsilon O(1)\right] .
\end{aligned}
$$

If there exists a positive number $K \leq k_{\lambda}\left(\lambda_{1}\right)$, such that (12) is still valid when replacing $k_{\lambda}\left(\lambda_{1}\right)$ by $K$, then, in particular, we have

$$
\begin{aligned}
\varepsilon \widetilde{I} & =\varepsilon \sum_{n=1}^{\infty} \sum_{m=1}^{\infty} \frac{\ln \left(U_{m} / V_{n}\right)}{U_{m}^{\lambda}-V_{n}^{\lambda}} \widetilde{a}_{m} \widetilde{b}_{n} \\
& <\varepsilon K\left[\sum_{m=1}^{\infty} \frac{U_{m}^{p\left(1-\lambda_{1}\right)-1}}{\mu_{m}^{p-1}} \widetilde{a}_{m}^{p}\right]^{\frac{1}{p}}\left[\sum_{n=1}^{\infty} \frac{V_{n}^{q\left(1-\lambda_{2}\right)-1}}{v_{n}^{q-1}} \widetilde{b}_{n}^{q}\right]^{\frac{1}{q}} .
\end{aligned}
$$


We obtain from the above results

$$
\left[\frac{\pi}{\lambda \sin \left(\pi \widetilde{\lambda}_{1} / \lambda\right)}\right]^{2}\left[1+o_{2}(1)-\varepsilon O(1)\right]<K\left(1+o_{1}(1)\right)^{\frac{1}{p}}\left(1+o_{2}(1)\right)^{\frac{1}{q}}
$$

and then it follows that $k_{\lambda}\left(\lambda_{1}\right) \leq K$ (for $\varepsilon \rightarrow 0^{+}$). Hence $K=k_{\lambda}\left(\lambda_{1}\right)$ is the best value of (12).

We conform that the constant factor $k_{\lambda}\left(\lambda_{1}\right)$ in (13) is the best possible. Otherwise we can get a contradiction by (16): that the constant factor in (12) is not the best value.

For $p>1$, setting

$$
\varphi(m):=\frac{U_{m}^{p\left(1-\lambda_{1}\right)-1}}{\mu_{m}^{p-1}}, \quad \psi(n):=\frac{V_{n}^{q\left(1-\lambda_{2}\right)-1}}{v_{n}^{q-1}} \quad(n, m \in \mathbf{N})
$$

then it follows that $[\psi(n)]^{1-p}=\frac{v_{n}}{V_{n}^{1-p \lambda_{2}}}$, and we define the real weighted normed function spaces as follows:

$$
\begin{aligned}
& l_{p, \varphi}:=\left\{a=\left\{a_{m}\right\}_{m=1}^{\infty} ;\|a\|_{p, \varphi}=\left\{\sum_{m=1}^{\infty} \frac{U_{m}^{p\left(1-\lambda_{1}\right)-1}}{\mu_{m}^{p-1}}\left|a_{m}\right|^{p}\right\}^{\frac{1}{p}}<\infty\right\}, \\
& l_{q, \psi}:=\left\{b=\left\{b_{n}\right\}_{n=1}^{\infty} ;\|b\|_{q, \psi}=\left\{\sum_{n=1}^{\infty} \frac{V_{n}^{q\left(1-\lambda_{2}\right)-1}}{v_{n}^{q-1}}\left|b_{n}\right|^{q}\right\}^{\frac{1}{q}}<\infty\right\}, \\
& l_{p, \psi 1-p}:=\left\{c=\left\{c_{n}\right\}_{n=1}^{\infty} ;\|c\|_{p, \psi} 1-p=\left\{\sum_{n=1}^{\infty} \frac{v_{n}}{V_{n}^{1-p \lambda_{2}}}\left|c_{n}\right|^{p}\right\}^{\frac{1}{p}}<\infty\right\} .
\end{aligned}
$$

For $a=\left\{a_{m}\right\}_{m=1}^{\infty} \in l_{p, \varphi}$, putting $h_{n}:=\sum_{m=1}^{\infty} \frac{\ln \left(U_{m} / V_{n}\right)}{U_{m}^{\lambda}-V_{n}^{\lambda}} a_{m}, h=\left\{h_{n}\right\}_{n=1}^{\infty}$, then it follows by (13) that $\|h\|_{p, \psi^{1-p}}<k_{\lambda}\left(\lambda_{1}\right)\|a\|_{p, \varphi}$, and $h \in l_{p, \psi^{1-p}}$.

Definition 1 Define a Hardy-Hilbert-type operator $T: l_{p, \varphi} \rightarrow l_{p, \psi^{1-p}}$ as follows: For $a_{m} \geq 0, a=\left\{a_{m}\right\}_{m=1}^{\infty} \in l_{p, \varphi}$, there exists a unique representation $T a=h \in l_{p, \psi^{1-p}}$. We define the following formal inner product of $T a$ and $b=\left\{b_{n}\right\}_{n=1}^{\infty} \in l_{q, \psi}\left(b_{n} \geq 0\right)$ as follows:

$$
(T a, b):=\sum_{n=1}^{\infty} \sum_{m=1}^{\infty} \frac{\ln \left(U_{m} / V_{n}\right)}{U_{m}^{\lambda}-V_{n}^{\lambda}} a_{m} b_{n}
$$

Hence (12) and (13) may be rewritten in terms of the following operator expressions:

$$
\begin{aligned}
& (T a, b)<k_{\lambda}\left(\lambda_{1}\right)\|a\|_{p, \varphi}\|b\|_{q, \psi}, \\
& \|T a\|_{p, \psi^{1-p}}<k_{\lambda}\left(\lambda_{1}\right)\|a\|_{p, \varphi} .
\end{aligned}
$$

It follows that the operator $T$ is bounded with

$$
\|T\|:=\sup _{a(\neq \theta) \in l_{p, \varphi}} \frac{\|T a\|_{p, \psi^{1-p}}}{\|a\|_{p, \varphi}} \leq k_{\lambda}\left(\lambda_{1}\right)
$$


Since the constant factor $k_{\lambda}\left(\lambda_{1}\right)$ in (19) is the best possible, we have

$$
\|T\|=k_{\lambda}\left(\lambda_{1}\right)=\left[\frac{\pi}{\lambda \sin \left(\lambda_{1} \pi / \lambda\right)}\right]^{2}
$$

\section{Some reverses}

We set $\widetilde{\varphi}(m):=\left(1-\theta_{1}\left(\lambda_{2}, m\right)\right) \frac{u_{m}^{p\left(1-\lambda_{1}\right)-1}}{\mu_{m}^{p-1}}, \widetilde{\psi}(n):=\left(1-\theta_{2}\left(\lambda_{1}, m\right)\right) \frac{V_{n}^{q\left(1-\lambda_{2}\right)-1}}{v_{n}^{q-1}}(n, m \in \mathbf{N})$. For $0<$ $p<1$ or $p<0$, we still use the formal symbol of the norm in this part for convenience.

Theorem 3 Suppose that $0<p<1,\left\{\mu_{m}\right\}_{m=1}^{\infty}$ and $\left\{v_{n}\right\}_{n=1}^{\infty}$ are decreasing positive sequences, and $U(\infty)=V(\infty)=\infty$, then we have the following equivalent inequalities:

$$
\begin{aligned}
I & =\sum_{n=1}^{\infty} \sum_{m=1}^{\infty} \frac{\ln \left(U_{m} / V_{n}\right)}{U_{m}^{\lambda}-V_{n}^{\lambda}} a_{m} b_{n}>\left[\frac{\pi}{\lambda \sin \left(\pi \lambda_{1} / \lambda\right)}\right]^{2}\|a\|_{p, \tilde{\varphi}}\|b\|_{q, \psi}, \\
J & =\left\{\sum_{n=1}^{\infty} \frac{v_{n}}{V_{n}^{1-p \lambda_{2}}}\left(\sum_{m=1}^{\infty} \frac{\ln \left(U_{m} / V_{n}\right)}{U_{m}^{\lambda}-V_{n}^{\lambda}} a_{m}\right)^{p}\right\}^{\frac{1}{p}} \\
& >\left[\frac{\pi}{\lambda \sin \left(\pi \lambda_{1} / \lambda\right)}\right]^{2}\|a\|_{p, \tilde{\varphi}}
\end{aligned}
$$

where the constant factor $\left[\frac{\pi}{\lambda \sin \left(\pi \lambda_{1} / \lambda\right)}\right]^{2}$ is the best possible.

Proof By the reverse Hölder inequality with weight (cf. [14]) and (7), we obtain the reverse forms of (14) and (15). It follows that (22) is valid by (8). Using the reverse Hölder inequality (cf. [14]), we find

$$
I=\sum_{n=1}^{\infty}\left[\frac{v_{n}^{1 / p}}{V_{n}^{\frac{1}{p}-\lambda_{2}}} \sum_{m=1}^{\infty} \frac{\ln \left(U_{m} / V_{n}\right)}{U_{m}^{\lambda}-V_{n}^{\lambda}} a_{m}\right]\left[\frac{V_{n}^{\frac{1}{p}-\lambda_{2}}}{v_{n}^{1 / p}} b_{n}\right] \geq J\|b\|_{q, \psi}
$$

Hence (21) is valid by using (22). Setting

$$
b_{n}=\frac{v_{n}}{V_{n}^{1-p \lambda_{2}}}\left[\sum_{m=1}^{\infty} \frac{\ln \left(U_{m} / V_{n}\right)}{U_{m}^{\lambda}-V_{n}^{\lambda}} a_{m}\right]^{p-1}, \quad n \in \mathbf{N}
$$

then we have $J=\left[\sum_{n=1}^{\infty} \frac{V_{n}^{q\left(1-\lambda_{2}\right)-1}}{v_{n}^{q-1}} b_{n}^{q}\right]^{1 / p}$. Assume that (21) is valid. By the reverse of (15), it follows that $J>0$. If $J=\infty$, then (22) is trivially valid. If $0<J<\infty$, then we find

$$
\begin{aligned}
& \sum_{n=1}^{\infty} \frac{V_{n}^{q\left(1-\lambda_{2}\right)-1}}{v_{n}^{q-1}} b_{n}^{q}=J^{p}=I>k_{\lambda}\left(\lambda_{1}\right)\|a\|_{p, \widetilde{\varphi}}\left[\sum_{n=1}^{\infty} \frac{V_{n}^{q\left(1-\lambda_{2}\right)-1}}{v_{n}^{q-1}} b_{n}^{q}\right]^{\frac{1}{q}}, \\
& J=\left[\sum_{n=1}^{\infty} \frac{V_{n}^{q\left(1-\lambda_{2}\right)-1}}{v_{n}^{q-1}} b_{n}^{q}\right]^{1 / p}>k_{\lambda}\left(\lambda_{1}\right)\|a\|_{p, \widetilde{\varphi}}
\end{aligned}
$$

Hence (22) is valid, which is equivalent to (21). 
For $0<\varepsilon<p \lambda_{1}$, we set $\tilde{\lambda}_{1}=\lambda_{1}-\frac{\varepsilon}{p}(\in(0,1)), \tilde{\lambda}_{2}=\lambda_{2}+\frac{\varepsilon}{p}(>0), \tilde{a}_{m}=U_{m}^{\tilde{\lambda}_{1}-1} \mu_{m}, \tilde{b}_{n}=$ $V_{n}^{\tilde{\lambda}_{2}-\varepsilon-1} v_{n}$. By (10), (11), and (7), in view of Remark 1 , we find

$$
\begin{aligned}
& \sum_{m=1}^{\infty}\left(1-\theta_{1}\left(\lambda_{2}, m\right)\right) \frac{U_{m}^{p\left(1-\lambda_{1}\right)-1}}{\mu_{m}^{p-1}} \widetilde{a}_{m}^{p} \\
& =\sum_{m=1}^{\infty}\left(1-O\left(\frac{1}{U_{m}^{\lambda_{2} / 2}}\right)\right) \frac{\mu_{m}}{U_{m}^{1+\varepsilon}} \\
& =\sum_{m=1}^{\infty} \frac{\mu_{m}}{U_{m}^{1+\varepsilon}}-\sum_{m=1}^{\infty} O\left(\frac{\mu_{m}}{U_{m}^{1+\varepsilon+\left(\lambda_{2} / 2\right)}}\right)=\frac{1}{\varepsilon}\left(1+o_{1}(1)-\varepsilon O(1)\right), \\
& \sum_{n=1}^{\infty} \frac{V_{n}^{q\left(1-\lambda_{2}\right)-1}}{v_{n}^{q-1}} \tilde{b}_{n}^{q}=\sum_{n=1}^{\infty} \frac{v_{n}}{V_{n}^{1+\varepsilon}}=\frac{1}{\varepsilon}\left(1+o_{2}(1)\right), \\
& \widetilde{I}=\sum_{n=1}^{\infty} \sum_{m=1}^{\infty} \frac{\ln \left(U_{m} / V_{n}\right)}{U_{m}^{\lambda}-V_{n}^{\lambda}} \widetilde{a}_{m} \widetilde{b}_{n}=\sum_{n=1}^{\infty}\left[\sum_{m=1}^{\infty} \frac{\ln \left(U_{m} / V_{n}\right)}{U_{m}^{\lambda}-V_{n}^{\lambda}} \frac{V_{n}^{\tilde{\lambda}_{2}} \mu_{m}}{U_{m}^{1-\widetilde{\lambda}_{1}}}\right] \frac{v_{n}}{V_{n}^{\varepsilon+1}} \\
& =\sum_{n=1}^{\infty} \varpi\left(\tilde{\lambda}_{1}, n\right) \frac{v_{n}}{V_{n}^{\varepsilon+1}}<k_{\lambda}\left(\widetilde{\lambda}_{1}\right) \sum_{n=1}^{\infty} \frac{v_{n}}{V_{n}^{\varepsilon+1}} \\
& =\frac{1}{\varepsilon}\left[\frac{\pi}{\lambda \sin \left(\pi \tilde{\lambda}_{1} / \lambda\right)}\right]^{2}\left(1+o_{2}(1)\right) \text {. }
\end{aligned}
$$

If there exists a positive number $K \geq k_{\lambda}\left(\lambda_{1}\right)$, such that (21) is still valid when replacing $k_{\lambda}\left(\lambda_{1}\right)$ by $K$, then in particular, we have

$$
\begin{aligned}
\varepsilon \widetilde{I} & =\varepsilon \sum_{n=1}^{\infty} \sum_{m=1}^{\infty} \frac{\ln \left(U_{m} / V_{n}\right)}{U_{m}^{\lambda}-V_{n}^{\lambda}} \widetilde{a}_{m} \widetilde{b}_{n} \\
& >\varepsilon K\left[\sum_{m=1}^{\infty}\left(1-\theta_{1}\left(\lambda_{2}, m\right)\right) \frac{U_{m}^{p\left(1-\lambda_{1}\right)-1}}{\mu_{m}^{p-1}} \widetilde{a}_{m}^{p}\right]^{\frac{1}{p}}\left[\sum_{n=1}^{\infty} \frac{V_{n}^{q\left(1-\lambda_{2}\right)-1}}{v_{n}^{q-1}} \widetilde{b}_{n}^{q}\right]^{\frac{1}{q}} .
\end{aligned}
$$

We obtain from the above results that

$$
\left[\frac{\pi}{\lambda \sin \left(\pi \widetilde{\lambda}_{1} / \lambda\right)}\right]^{2}\left(1+o_{2}(1)\right)>K\left(1+o_{1}(1)-\varepsilon O(1)\right)^{\frac{1}{p}}\left(1+o_{2}(1)\right)^{\frac{1}{q}}
$$

and then $k_{\lambda}\left(\lambda_{1}\right) \geq K$ (for $\varepsilon \rightarrow 0^{+}$). Hence $K=k_{\lambda}\left(\lambda_{1}\right)$ is the best value of (21).

We conform that the constant factor $k_{\lambda}\left(\lambda_{1}\right)$ in (22) is the best possible. Otherwise we can get a contradiction by (23): that the constant factor in (21) is not the best value.

Theorem 4 Suppose that $p<0,\left\{\mu_{m}\right\}_{m=1}^{\infty}$ and $\left\{v_{n}\right\}_{n=1}^{\infty}$ are decreasing positive sequences, and $U(\infty)=V(\infty)=\infty$, then we have the following equivalent inequalities:

$$
I=\sum_{n=1}^{\infty} \sum_{m=1}^{\infty} \frac{\ln \left(U_{m} / V_{n}\right)}{U_{m}^{\lambda}-V_{n}^{\lambda}} a_{m} b_{n}>\left[\frac{\pi}{\lambda \sin \left(\pi \lambda_{1} / \lambda\right)}\right]^{2}\|a\|_{p, \varphi}\|b\|_{q, \tilde{\psi}}
$$




$$
\begin{aligned}
J_{1} & =\left\{\sum_{n=1}^{\infty} \frac{\left(1-\theta_{2}\left(\lambda_{1}, n\right)\right)^{1-p} v_{n}}{V_{n}^{1-p \lambda_{2}}}\left(\sum_{m=1}^{\infty} \frac{\ln \left(U_{m} / V_{n}\right)}{U_{m}^{\lambda}-V_{n}^{\lambda}} a_{m}\right)^{p}\right\}^{\frac{1}{p}} \\
& >\left[\frac{\pi}{\lambda \sin \left(\pi \lambda_{1} / \lambda\right)}\right]^{2}\|a\|_{p, \varphi},
\end{aligned}
$$

where the constant factor $\left[\frac{\pi}{\lambda \sin \left(\pi \lambda_{1} / \lambda\right)}\right]^{2}$ is the best possible.

Proof Using the same way of obtaining (14) and (15), by the reverse Hölder inequality with weight and (9), we have

$$
J_{1}>\left(k_{\lambda}\left(\lambda_{1}\right)\right)^{\frac{1}{q}}\left[\sum_{m=1}^{\infty} \omega\left(\lambda_{2}, m\right) \frac{U_{m}^{p\left(1-\lambda_{1}\right)-1}}{\mu_{m}^{p-1}} a_{m}^{p}\right]^{\frac{1}{p}},
$$

then we obtain (25) by (6). Using the reverse Hölder inequality, we have

$$
\begin{aligned}
I= & \sum_{n=1}^{\infty}\left[\frac{\left(1-\theta_{2}\left(\lambda_{1}, n\right)\right)^{-\frac{1}{q}} v_{n}^{1 / p}}{V_{n}^{\frac{1}{p}-\lambda_{2}}} \sum_{m=1}^{\infty} \frac{\ln \left(U_{m} / V_{n}\right)}{U_{m}^{\lambda}-V_{n}^{\lambda}} a_{m}\right] \\
& \times\left[\left(1-\theta_{2}\left(\lambda_{1}, n\right)\right)^{\frac{1}{q}} \frac{V_{n}^{\frac{1}{p}-\lambda_{2}}}{v_{n}^{1 / p}} b_{n}\right] \\
\geq & J_{1}\|b\|_{q, \tilde{\psi}} .
\end{aligned}
$$

Hence (24) is valid by (25). Assuming that (24) is valid, setting

$$
b_{n}=\frac{\left(1-\theta_{2}\left(\lambda_{1}, n\right)\right)^{1-p} v_{n}}{V_{n}^{1-p \lambda_{2}}}\left[\sum_{m=1}^{\infty} \frac{\ln \left(U_{m} / V_{n}\right)}{U_{m}^{\lambda}-V_{n}^{\lambda}} a_{m}\right]^{p-1}, \quad n \in \mathbf{N},
$$

we find

$$
J_{1}=\left[\sum_{n=1}^{\infty}\left(1-\theta_{2}\left(\lambda_{1}, n\right)\right) \frac{V_{n}^{q\left(1-\lambda_{2}\right)-1}}{v_{n}^{q-1}} b_{n}^{q}\right]^{1 / p} .
$$

It follows that $J_{1}>0$ by (26). If $J_{1}=\infty$, then (25) is trivially valid. If $0<J_{1}<\infty$, then we find

$$
\begin{aligned}
& \sum_{n=1}^{\infty}\left(1-\theta_{2}\left(\lambda_{1}, n\right)\right) \frac{V_{n}^{q\left(1-\lambda_{2}\right)-1}}{v_{n}^{q-1}} b_{n}^{q}=J_{1}^{p}=I \\
& \qquad k_{\lambda}\left(\lambda_{1}\right)\|a\|_{p, \varphi}\left[\sum_{n=1}^{\infty}\left(1-\theta_{2}\left(\lambda_{1}, n\right)\right) \frac{V_{n}^{q\left(1-\lambda_{2}\right)-1}}{v_{n}^{q-1}} b_{n}^{q}\right]^{\frac{1}{q}}, \\
& J_{1}=\left[\sum_{n=1}^{\infty}\left(1-\theta_{2}\left(\lambda_{1}, n\right)\right) \frac{V_{n}^{q\left(1-\lambda_{2}\right)-1}}{v_{n}^{q-1}} b_{n}^{q}\right]^{1 / p}>k_{\lambda}\left(\lambda_{1}\right)\|a\|_{p, \varphi} .
\end{aligned}
$$

Hence (25) is valid, which is equivalent to (24). 
For $0<\varepsilon<q \lambda_{2}$, we set $\tilde{\lambda}_{1}=\lambda_{1}+\frac{\varepsilon}{q}(>0), \tilde{\lambda}_{2}=\lambda_{2}-\frac{\varepsilon}{q}(\in(0,1)), \widetilde{a}_{m}=U_{m}^{\widetilde{\lambda}_{1}-\varepsilon-1} \mu_{m}, \widetilde{b}_{n}=$ $V_{n}^{\widetilde{\lambda}_{2}-1} v_{n}$. By (10), (11), and (6), in view of Remark 1, we have

$$
\begin{aligned}
& \sum_{m=1}^{\infty} \frac{U_{m}^{p\left(1-\lambda_{1}\right)-1}}{\mu_{m}^{p-1}} \widetilde{a}_{m}^{p}=\sum_{m=1}^{\infty} \frac{\mu_{m}}{U_{m}^{1+\varepsilon}}=\frac{1}{\varepsilon}\left(1+o_{1}(1)\right) \\
& \sum_{n=1}^{\infty}\left(1-\theta_{2}\left(\lambda_{1}, n\right)\right) \frac{V_{n}^{q\left(1-\lambda_{2}\right)-1}}{v_{n}^{q-1}} \widetilde{b}_{n}^{q} \\
& \quad=\sum_{n=1}^{\infty}\left(1-O\left(\frac{1}{V_{n}^{\lambda_{1} / 2}}\right)\right) \frac{v_{n}}{V_{n}^{1+\varepsilon}} \\
& \quad=\frac{1}{\varepsilon}\left(1+o_{2}(1)-\varepsilon O(1)\right), \\
& \widetilde{I}=\sum_{n=1}^{\infty} \sum_{m=1}^{\infty} \frac{\ln \left(U_{m} / V_{n}\right)}{U_{m}^{\lambda}-V_{n}^{\lambda}} \widetilde{a}_{m} \widetilde{b}_{n} \\
& =\sum_{m=1}^{\infty}\left[\sum_{n=1}^{\infty} \frac{\ln \left(U_{m} / V_{n}\right)}{U_{m}^{\lambda}-V_{n}^{\lambda}} \frac{U_{m}^{\lambda_{1}} v_{n}}{V_{n}^{1-\tilde{\lambda}_{2}}}\right] \frac{\mu_{m}}{U_{m}^{1+\varepsilon}} \\
& =\sum_{m=1}^{\infty} \varpi\left(\widetilde{\lambda}_{2}, m\right) \frac{\mu_{m}}{U_{m}^{1+\varepsilon}}<k_{\lambda}\left(\widetilde{\lambda}_{1}\right) \sum_{n=1}^{\infty} \frac{\mu_{m}}{U_{m}^{1+\varepsilon}} \\
& \quad=\frac{1}{\varepsilon}\left[\frac{\pi}{\lambda \sin \left(\pi \widetilde{\lambda}_{1} / \lambda\right)}\right]^{2}\left(1+o_{1}(1)\right) .
\end{aligned}
$$

If there exists a positive number $K \geq k_{\lambda}\left(\lambda_{1}\right)$, such that (24) is still valid as we replace $k_{\lambda}\left(\lambda_{1}\right)$ by $K$, then, in particular, we have

$$
\begin{aligned}
\varepsilon \widetilde{I} & =\varepsilon \sum_{n=1}^{\infty} \sum_{m=1}^{\infty} \frac{\ln \left(U_{m} / V_{n}\right)}{U_{m}^{\lambda}-V_{n}^{\lambda}} \widetilde{a}_{m} \widetilde{b}_{n} \\
& >\varepsilon K\left[\sum_{m=1}^{\infty} \frac{U_{m}^{p\left(1-\lambda_{1}\right)-1}}{\mu_{m}^{p-1}} \widetilde{a}_{m}^{p}\right]^{\frac{1}{p}}\left[\sum_{n=1}^{\infty}\left(1-\theta_{2}\left(\lambda_{1}, n\right)\right) \frac{V_{n}^{q\left(1-\lambda_{2}\right)-1}}{v_{n}^{q-1}} \widetilde{b}_{n}^{q}\right]^{\frac{1}{q}} .
\end{aligned}
$$

From the above results, we have

$$
\left[\frac{\pi}{\lambda \sin \left(\pi \widetilde{\lambda}_{1} / \lambda\right)}\right]^{2}\left(1+o_{1}(1)\right)>K\left(1+o_{1}(1)\right)^{\frac{1}{p}}\left(1+o_{2}(1)-\varepsilon O(1)\right)^{\frac{1}{q}} .
$$

It follows that $k_{\lambda}\left(\lambda_{1}\right) \geq K$ (for $\varepsilon \rightarrow 0^{+}$). Hence $K=k_{\lambda}\left(\lambda_{1}\right)$ is the best value of (24). We conform that the constant factor $k_{\lambda}\left(\lambda_{1}\right)$ in (25) is the best possible. Otherwise we can get a contradiction by (27): that the constant factor in (24) is not the best value.

Remark 2 For $\mu_{i}=v_{i}=1(i=1,2, \ldots),(12)$ reduces to (3); for $\lambda=1, \lambda_{1}=\frac{1}{q}, \lambda_{2}=\frac{1}{p}$, it follows by (12) that

$$
\sum_{n=1}^{\infty} \sum_{m=1}^{\infty} \frac{\ln \left(U_{m} / V_{n}\right)}{U_{m}-V_{n}} a_{m} b_{n}<\left[\frac{\pi}{\sin (\pi / p)}\right]^{2}\left[\sum_{m=1}^{\infty} \frac{1}{\mu_{m}^{p-1}} a_{m}^{p}\right]^{1 / p}\left[\sum_{n=1}^{\infty} \frac{1}{v_{n}^{q-1}} b_{n}^{q}\right]^{1 / q}
$$


for $\lambda=1, \lambda_{1}=\frac{1}{p}, \lambda_{2}=\frac{1}{q},(12)$ reduces to the dual form of (28) as follows:

$$
\sum_{n=1}^{\infty} \sum_{m=1}^{\infty} \frac{\ln \left(U_{m} / V_{n}\right)}{U_{m}-V_{n}} a_{m} b_{n}<\left[\frac{\pi}{\sin (\pi / p)}\right]^{2}\left[\sum_{m=1}^{\infty} \frac{U_{m}^{p-2}}{\mu_{m}^{p-1}} a_{m}^{p}\right]^{1 / p}\left[\sum_{n=1}^{\infty} \frac{V_{n}^{q-2}}{v_{n}^{q-1}} b_{n}^{q}\right]^{1 / q} .
$$

\section{Competing interests}

The author declares to have no competing interests.

\section{Author's contributions}

$\mathrm{QH}$ carried out the mathematical studies, sequenced alignment, drafted the manuscript, and performed the numerical analysis. The author read and approved the final manuscript.

\section{Acknowledgements}

This work is supported by the 2013 Knowledge Construction Special Foundation Item of Guangdong Institution of Higher Learning College and University (No. 2013KJCX0140).

Received: 29 July 2015 Accepted: 29 November 2015 Published online: 15 December 2015

\section{References}

1. Hardy, GH: Note on a theorem of Hilbert concerning series of positive terms. Proc. Lond. Math. Soc. (2) 23, xlv-xlvi (1925)

2. Hardy, GH, Littlewood, JE, Pólya, G: Inequalities. Cambridge University Press, Cambridge (1934)

3. Yang, BC: An extension of the Hilbert-type inequality and its reverse. J. Math. Inequal. 2(1), 139-149 (2008)

4. Yang, BC: Generalization of Hilbert's type inequality with best constant factor and its applications. J. Math. Res. Expo. 25(2), 341-346 (2005) (in Chinese)

5. Yang, BC: On a more accurate Hardy-Hilbert's type inequality and its applications. Acta Math. Sin. New Ser. 49(2), 363-368 (2006)

6. Yang, BC: On the norm of a Hilbert's type linear operator and applications. J. Math. Anal. Appl. 325, 529-541 (2007)

7. Yang, BC: On a new Hardy-Hilbert's type inequality with a parameter. Int. J. Math. Anal. 1, 123-131 (2007)

8. Wang, WH, Yang, BC: A strengthened Hardy-Hilbert's type inequality. Aust. J. Math. Anal. Appl. 2, 1-7 (2006)

9. Zhong, WY, Yang, BC: A reverse Hilbert's type integral inequality with some parameters and the equivalent forms. Pure Appl. Math. 24(2), 401-407 (2008)

10. He, B: On a bilateral Hilbert-type inequality with a homogeneous kernel of 0-degree. Kyungpook Math. J. 50, 307-314 (2010)

11. Xin, DM, Yang, BC: A basic Hilbert-type inequality. J. Math. 30(3), 554-560 (2010)

12. Yang, BC: The Norm of Operator and Hilbert-Type Inequalities. Science Press, Beijin (2009) (in Chinese)

13. Yang, BC: Discrete Hilbert-Type Inequalities. Bentham Science Publishers Ltd, Sharjah (2011)

14. Kuang, J: Applied Inequalities. Shangdong Science Technic Press, Jinan (2010) (in Chinese)

\section{Submit your manuscript to a SpringerOpen ${ }^{\circ}$ journal and benefit from:}

- Convenient online submission

Rigorous peer review

- Immediate publication on acceptance

- Open access: articles freely available online

- High visibility within the field

- Retaining the copyright to your article 